\title{
Global dimming and urbanization: did stronger negative SSR trends collocate with regions of population growth?
}

\author{
Adel Imamovic $^{1}$, Katsumasa Tanaka ${ }^{1,2}$, Doris Folini ${ }^{1}$, and Martin Wild ${ }^{1}$ \\ ${ }^{1}$ Institute for Atmospheric and Climate Science, ETH Zürich, Switzerland \\ ${ }^{2}$ National Institute for Environmental Studies (NIES), Tsukuba, Japan \\ Correspondence to: Adel Imamovic (adel.imamovic@env.ethz.ch)
}

Received: 31 July 2015 - Published in Atmos. Chem. Phys. Discuss.: 6 November 2015

Revised: 1 February 2016 - Accepted: 11 February 2016 - Published: 4 March 2016

\begin{abstract}
Global dimming refers to the decrease in surface solar radiation (SSR) observed from the 1960s to the 1980s at different measurement sites all around the world. It is under debate whether anthropogenic aerosols emitted from urban areas close to the measurement sites are mainly responsible for the dimming. In order to assess this urbanization impact on SSR, we use spatially explicit population density data of $0.08^{\circ}$ resolution to construct population indices (PI) at 157 high data quality sites. Our study extends previous population-based studies by incorporating distanceweighting as a simple aerosol diffusion model. We measured urbanization in the surrounding of a site as the PI change from 1960 to 1990 and found no negative correlation with the corresponding SSR trends from 1964 to 1989 for the 92 sites in Europe and Japan. For the 39 sites in China the correlation coefficients are significant at the $5 \%$ level and reach around -0.35 , while for the 26 remaining Asian, mostly Russian sites the correlation coefficients reach around -0.55 at the $1 \%$ significance level. Results are similar, when the absolute levels of PIs are taken as an indicator for urbanization. Our findings call into question the existence of an urbanization effect for the sites in Europe and Japan, while such an effect cannot be ruled out for the sites in Asia, especially in Russia.
\end{abstract}

\section{Introduction}

Surface solar radiation (SSR) is the sum of direct and diffuse solar radiation incident at the surface of the Earth. Systematic and widespread pyranometer-based measurements of SSR started in the mid-twentieth cen- tury and were compiled in databases such as the Global Energy Balance Archive (GEBA) at ETH Zürich (Gilgen and Ohmura, 1999). From the 1960s to the 1980s the SSR underwent a prominent negative trend which became known as the global dimming (Ohmura and Lang, 1989). It was first discovered at sites in Europe and later worldwide (Gilgen et al., 1998). The average SSR trend estimates (in $\mathrm{W} \mathrm{m}^{-2} \mathrm{decade}^{-1}$ ) for global land sites during that period range between -2.3 (Liepert, 2002) and -5.1 (Stanhill and Cohen, 2001). A more detailed overview of trend estimates can be found in Table 1 of the review by Wild (2009). The negative trends started to reverse generally in the 1980s and marked the beginning of a global brightening (Wild et al., 2005). This study focuses on the time period from the 1960 s to the 1980s, which we refer to as the dimming period.

It is not completely understood what caused the global dimming. Apart from an increasing cloudiness, one of the most prominent explanations is an increasing aerosol optical depth (AOD) caused by rising global anthropogenic aerosol emissions from the 1960s to the 1980s. Simultaneous and systematic measurements of SSR/AOD or SSR/cloudiness during the dimming period are scarce. A few studies available deliver regionally dependent findings: for example Ohmura (2009) showed for five sites in Europe and Japan with simultaneous measurement of SSR and AOD that the aerosol direct and indirect effects are equally responsible for changing SSR. Norris and Wild (2007) used clear sky data and found that the cloud cover effects are insignificant for the dimming over Europe. In contrast to the sites in Europe, Liley (2009) used lidar-based measurements and showed that the AOD contributions to the dimming in New Zealand are insignificant; from a record of sunshine hours an increasing 
cloudiness was inferred and shown to be more consistent with the observed SSR pattern. This consistency was also found in Japan (Stanhill and Cohen, 2008).

Speculations have arisen that global dimming is only due to local pollution sources (i.e. a growing city or an industry plant) near the measurement sites and not a largerscale phenomenon (Alpert and Kishcha, 2008). This idea of nearby settlements affecting atmospheric measurements is commonly referred to as the urbanization effect. However, even if aerosols had a significant impact on SSR trends, it is not clear how far the effect persists, i.e. whether the proximity to an aerosol emission source determined the dimming trends. Due to the lack of fine-scale emission data, an estimate of urbanization was restricted to the use of proxy data, from which aerosol emissions could be inferred. Early approaches therefore used data on population to assess potential urbanization impacts on single sites, implicitly assuming that more people lead to more emissions and hence a stronger urbanization impact.

Gilgen et al. (1998) found the sites with the strongest dimming to be concentrated within the populated regions of the northern hemispheric mid-latitudes. Stanhill and Cohen (2001) found no significant correlation between the SSR measured at 854 sites in the years 1958, 1965, 1975, 1985, and 1992 and the corresponding population densities in the one degree $(\simeq 100 \mathrm{~km})$ grid surrounding these sites in the year 2000. Alpert and Kishcha (2008) calculated the SSR trends 1964-1989 for 317 sites worldwide and compared them against the respective population density in the one degree cell surrounding the measurement site in the year 2000. They found a significant dimming only in the group of GEBA sites surrounded by a cell with a population density greater than $10 \mathrm{~km}^{-2}$ and a significant correlation between the strength of dimming and the population density in the year 2000. The group of sites in rural areas was found to have undergone an insignificant change in SSR. A more recent, but alternative approach by Wang et al. (2014) using night-time light data of the year 2000 came to opposite conclusions. Their analysis of 105 pairs of urban-rural sites that were less than two degrees $(\simeq 200 \mathrm{~km})$ apart showed that the geographic proximity to an urban area had a small impact on both mean and trend of SSR.

The role of urbanization in affecting SSR during the global dimming period has remained an open question. In particular, it is not clear whether or not the population growth in the surroundings of a site adequately explains the global dimming. This study tackles this question by using a finer spatially explicit population data set $(\Delta x \simeq 10 \mathrm{~km})$ to devise a population index (PI). The PI of a site is the weighted mean of the population density within a radius of $500 \mathrm{~km}$ from the site (Sect. 2). We extend the previous approaches that used unweighted data on population (Stanhill and Cohen, 2001; Alpert and Kishcha, 2008) in the following ways. The SSR trends from 1964 to 1989 of 157 high-quality GEBA sites in Asia, Europe, and Japan are compared against (a) the PI

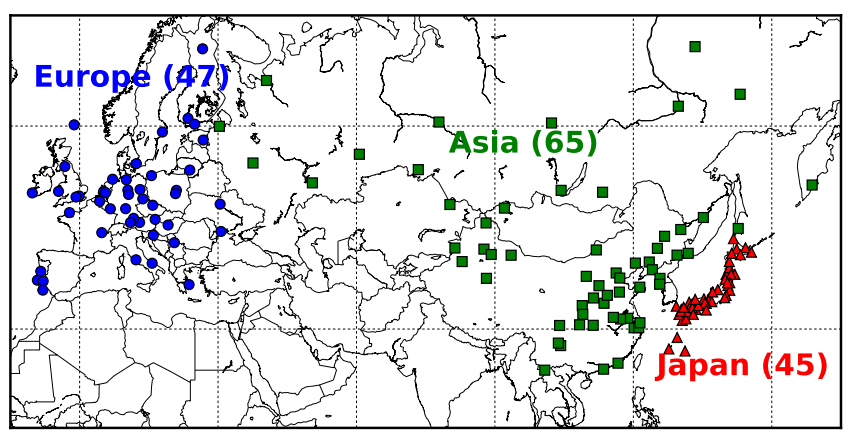

Figure 1. Distribution of the 157 GEBA sites analysed in this study. The group "Europe" consists of 47 sites (blue circles) and "Japan" consists of 45 sites (red triangles). The 39 Chinese, 22 Russian and 4 remaining Asian sites were aggregated into the "Asia" group (green squares).

changes from 1960 to 1990 , (b) the PI in 2000, and (c) the unweighted population density in the $0.08^{\circ}$ cell containing the site in the year 2000. The third approach is methodologically consistent with previous studies (Alpert and Kishcha, 2008; Stanhill and Cohen, 2001), as argued in Sect. 3. In doing so, we attempt to answer the question whether the sites considered in this study show a correlation between SSR trends and PI changes from 1960 to 1990 or a correlation between SSR trends and absolute levels of PI in the year 2000. With our analysis, we aim to contribute to the debate on whether global dimming is influenced by urbanization.

\section{Data and methods}

\subsection{Surface solar radiation}

Data on annual average SSR since the 1960s were taken from the GEBA. Measurement error estimates for annual mean SSR are $2 \%$ (Gilgen and Ohmura, 1999). We selected the same high-quality sites as used in the model data comparison by Skeie et al. (2011) and retained sites with at least 15 SSR annual averages in each 20-year time frame from 1960 to 1990 . This was fulfilled by 39 sites in China, 47 sites in Europe, 45 sites in Japan, 22 sites in Russia, and four sites in North Korea, Macau, and Mongolia. The latter four sites were aggregated with the Chinese and Russian sites into the group "Asia". The geographic distribution and the grouping of the 157 sites are shown in Fig. 1. For every GEBA site, the SSR trend for the period 1964-1989, i.e. the same period as used by Alpert and Kishcha (2008), was estimated using a bi-square regression.

\subsection{Population data and the population index}

We used the global population density data of the History Database of the Global Environment (HYDE), which is available at a spatial resolution of approximately $0.08^{\circ}(\simeq 10 \mathrm{~km})$ 

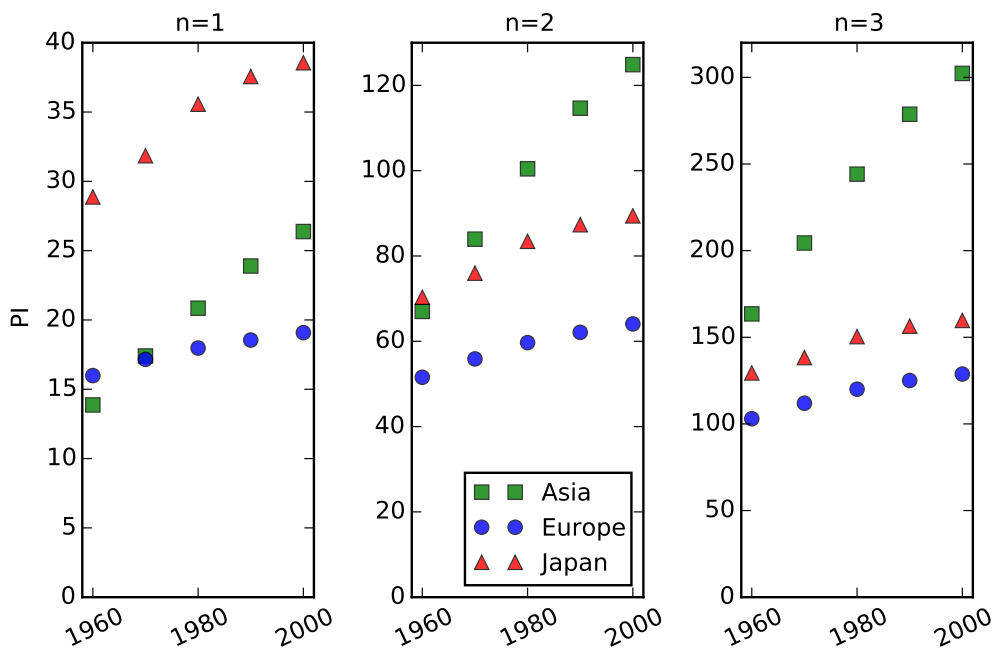

Figure 2. Evolutions of the region-averaged PIs of the sites in Asia, Europe and Japan for the scaling parameters $n=1,2$ and 3 (from left to right).

and a temporal resolution of 10 years for the years $t=1960$, 1970, 1980, 1990, and 2000. Error estimates for population density per grid cell are $\pm 1 \%$ (Klein Goldewijk et al., 2010).

In order to compare the surroundings of different GEBA sites, we converted the two dimensional population distribution around single sites into PI values. The PI is the weighted mean of the population density distribution within a radius of $500 \mathrm{~km}$ from the site: the weights are $w_{n}(s, y)=1 / d(s, y)^{n}$ i.e. the inverse of the distance between the measurement site and the population density cell under consideration to the $n$th power. Rationales for the choice of $n$ values for this study are explained further below in the text. The distance between a GEBA site at $s=\left[\theta_{s}, \varphi_{s}\right]$ and the centre of a nearby cell at $y=\left[\theta_{y}, \varphi_{y}\right]$ with population density $\rho(y, t)$ is denoted as $d(s, y)$. The distance is a function of the respective spherical coordinates $\theta_{s}, \varphi_{s}, \theta_{y}$, and $\varphi_{y}$ and can be looked up in mathematical handbooks.

If $1 / d(s, y)^{n}$ is used for the weighting of the population density cell that contains the site, an artificially large weight will result for cells whose centre is very close to the measurement site. For consistency the population density cell that contains the measurement site is not weighted with the inverse of the physical distance between the site and the centre of the cell to the $n$th power $1 / d(s, y)^{n}$ but with the inverse of the spatial extent $l$ of the cell to the $n$th power $1 / l^{n}$ instead. For the spatial extent $l$ of a population density cell we take the square root of the area of the cell. For cells in the mid-latitudes $l$ is approximately $7 \mathrm{~km}$.

We define the PI of a GEBA site $s$ at the time $t$ as the sum of all weighted population density grid cells within the $R=500 \mathrm{~km}$ surrounding of the site

$\mathrm{PI}(s, t)=\eta \sum_{y: d(s, y) \leq R} \frac{\rho(y, t)}{d(s, y)^{n}}$.
With the following definition of the normalization factor $\eta$ :

$$
\eta=\frac{1}{\rho_{0} \cdot \sum_{y: d(s, y) \leq R \frac{1}{d(s, y)^{n}}}}
$$

the PI becomes dimensionless and equals one for a fictitious site surrounded by a constant population density of $\rho_{0}=10 \mathrm{~km}^{-2}$. The value $R=500 \mathrm{~km}$ is chosen to reflect the finite lifetime and hence finite diffusion distance of aerosols and is obtained if one assumes a typical lifetime of 2 days at an average diffusion speed of $v=3 \mathrm{~m} \mathrm{~s}^{-1}$. We repeated the analysis for PIs with $R=750 \mathrm{~km}$ and $R=1000 \mathrm{~km}$ and found no significant differences in the results.

The weighting $1 / d(s, y)^{n}$ mimics a simple aerosol transport and diffusion model. By setting $n=0$ one assumes undiluted transport of all aerosols to the site. For $n=3$ pure diffusion of a tracer in three-dimensional space is assumed. Given these two bounds for $n$, we used the set $n=1,2,3$ to calculate PIs. Note that for small $n$, all cells in the surrounding of the GEBA contribute almost equally to the PI, as they receive almost equal weight. This can be seen mathematically as $w_{n}$ approaches unity for decreasing $n$. More precisely, a small $n$ $(\simeq 0)$ together with a small $R$ corresponds to previous studies that only used the unweighted population density in the one degree cell containing the site (Alpert and Kishcha, 2008). For increasing $n$ the weight factors converge to a $\delta$-function (which is zero everywhere except for the cell that contains the site), the PI for large $n$ carries mainly information on the closest settlements. The scaling parameter $n$ is thus a control parameter, that enables us to test the relative importance of aerosol travel distances. 

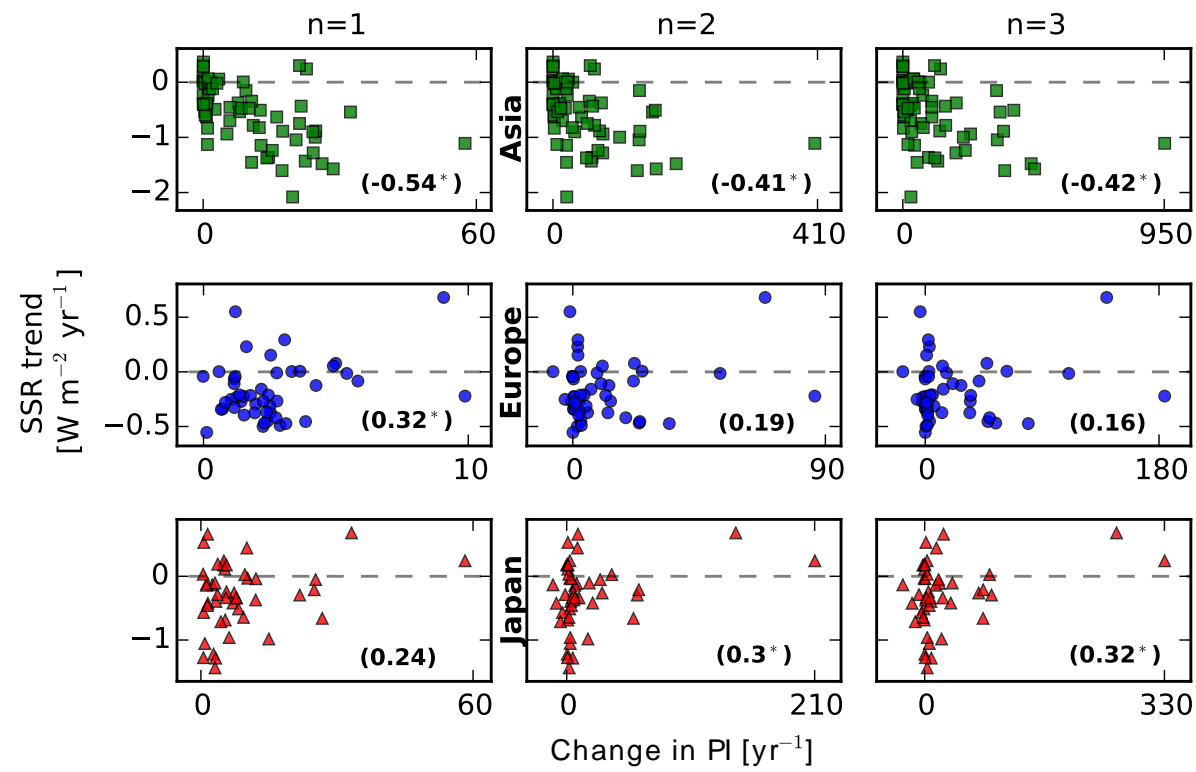

Figure 3. PI Change from 1960 to 1990 of the sites vs. the SSR trend from 1964 to 1989 for the three regions (top to bottom) and for the three scaling parameters $n=1,2,3$ (left to right). The correlation coefficients are indicated within the panels. The * indicates a significant correlation at the $5 \%$ level. The dashed line separates positive and negative SSR trends.

\section{Results}

For each GEBA site analysed in this study the PIs for $n=1,2,3$ and for the years $t=1960,1970,1980,1990$, and 2000 were calculated. The PIs of the GEBA sites averaged within the three regions Asia, Europe, and Japan generally increased from 1960 to 2000 for $n=1,2$, and 3 (Fig. 2). The 65 sites in Asia showed an average PI increase of $85 \%$ from 1960 to 2000. The average increase in PI for the sites in Japan is $20-30 \%$, while the increase was slightly smaller for the European sites. The European sites had both the smallest average increase in PI and the lowest absolute PI (except in 1960 for $n=1$ ). The average increase of PI of the sites in Europe and in Japan was similar for $n=2,3$ (similar slopes), while the absolute average PI was greater for the Japanese sites.

We investigated the impact of urbanization on SSR trends during the dimming period by calculating the correlation coefficients for SSR trends from 1964 to 1989 vs. the PI change from 1960 to 1990 (Fig. 3), and vs. the absolute PI in the year 2000 (Fig. 4).

\subsection{Europe and Japan}

The results for the sites in Europe and Japan are discussed together since they show similar features (second and third row in Fig. 3). The European sites underwent the weakest decrease in SSR (over the time period from 1964 to 1989) with a group average of $-0.17 \mathrm{~W} \mathrm{~m}^{-2} \mathrm{yr}^{-1}$. The largest decrease measured at a site in the European group was $-0.56 \mathrm{~W} \mathrm{~m}^{-2} \mathrm{yr}^{-1}$. The average decrease in SSR for the group of Japanese GEBA sites was $-0.30 \mathrm{~W} \mathrm{~m}^{-2} \mathrm{yr}^{-1}$ The maximum dimming in the group of Japanese sites was $-1.44 \mathrm{~W} \mathrm{~m}^{-2} \mathrm{yr}^{-1}$. The correlations between SSR trends and PI changes (Fig. 3) for the sites in Europe and Japan are positive and smaller than 0.33 for any choice of the scaling parameter $n$. It is important to note that the correlation coefficients are not negative, as would be expected from an urbanization impact: SSR trends are actually less negative for larger PI change. They are significant at the 5\% level only for the $n=1$ case in Europe, and for the $n=2,3$ cases in Japan. As shown in Fig. 4 similar correlation coefficients as in Fig. 3 emerge, when we take the PIs of the sites in year 2000 instead of the change in PI from 1960 to 1990. We obtained positive correlation coefficients at a significance level of $5 \%$, except for Japan for $n=1$.

For the 47 European and 45 Japanese sites considered in this study, the SSR trends are not well explained by the respective change in population or the absolute population in 2000.

\subsection{China and Russia}

The largest group-averaged SSR trends $\left(-0.55 \mathrm{~W} \mathrm{~m}^{-2} \mathrm{yr}^{-1}\right)$ and the largest changes in PI were reached at the GEBA sites in Asia. Figure 3 shows the SSR trends and the PI changes of the sites. Correlation coefficients for the changes in PI vs. the SSR trends are $-0.54,-0.41$, and -0.42 for $n=1,2$ and 3 , respectively, as shown in the first row of plots in Fig. 3. We obtain quantitatively similar correlation coefficients when we compare the SSR trends against the PI in the year 2000, instead of the PI change from 1960 to 1990 (Fig. 4). Note 

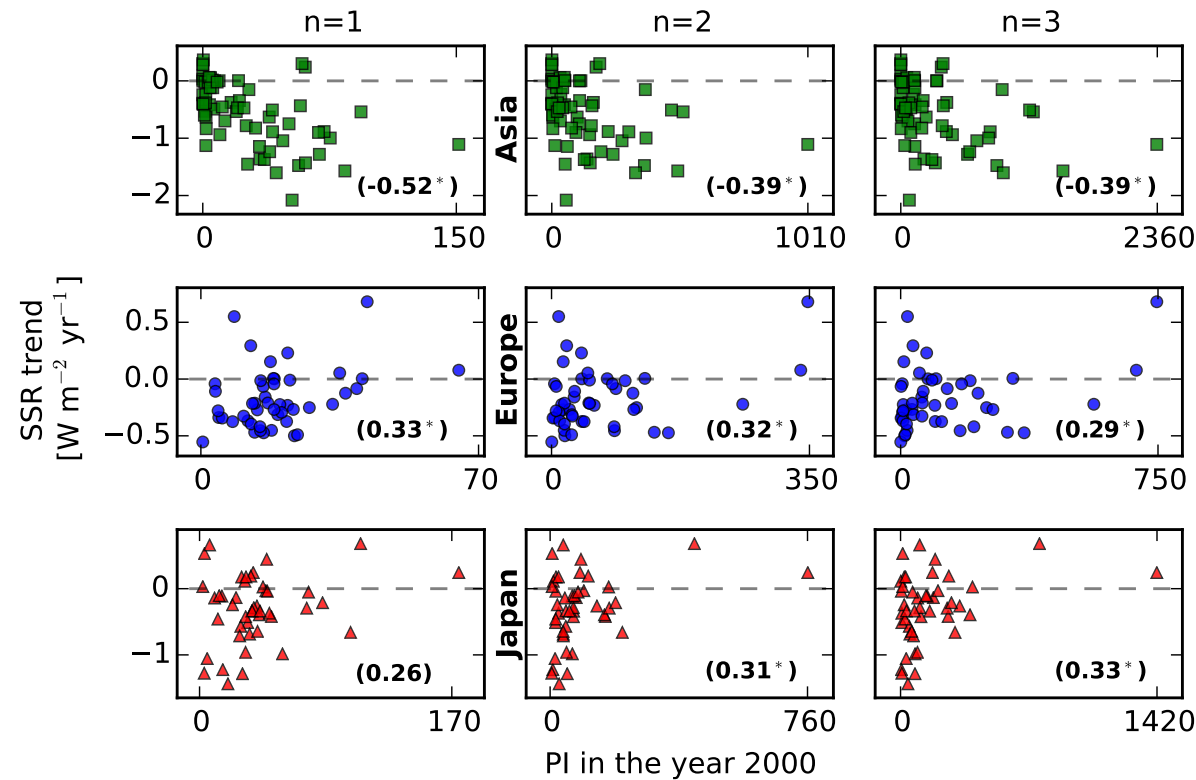

Figure 4. Same as in Fig. 3, but for the absolute PI of the sites in the year 2000 instead of the PI change from 1960-1990.

that the largest dimming of approximately $-2.0 \mathrm{~W} \mathrm{~m}^{-2} \mathrm{yr}^{-1}$ (lowest point in row 1 of Figs. 3 and 4) does not coincide with the site that exhibited the largest PI change or the largest PI in the year 2000. The correlation coefficients for the 30 right-most points in Fig. 3, i.e. the sites that underwent the greatest increase in PI, and correlation coefficients for the 30 left-most points, i.e. the sites with the smallest increase in PI, are both around $\rho=-0.30$.

We split the group of the 65 Asian sites further into the 39 Chinese sites and 26 non-Chinese, mostly Russian (22) GEBA sites (Fig. 5) and repeated the above analysis. As above, the correlation coefficients in these subgroups are quantitatively the same whether one takes the PI change from 1960 to 1990 or the PI in the year 2000 as an indicator for urbanization. The correlation coefficients for the non-Chinese sites are significant at the $1 \%$ level and range between -0.5 and -0.63 , while for the Chinese sites the correlation coefficients range between -0.33 and -0.35 and are significant at the $5 \%$ level.

Similar correlation coefficients for the sites in Asia, Europe and Japan (as documented above) are found when the unweighted population density in the year 2000 in the $0.08^{\circ}$ cell surrounding the GEBA site is compared against the SSR trends. Figure 6 shows that these correlation coefficients are very close to the corresponding correlation coefficients when the $n=3$ PIs in the year 2000 are used instead (third column in Fig. 4). An explanation for this can be found in Sect. 2.

\section{Discussions and conclusions}

Some studies argued that the observed negative SSR trends during the global dimming period from the 1960s to the 1980 s are restricted to urban areas. In order to assess the urbanization impact on SSR, previous studies used spatially explicit population density data in the one degree cell surrounding the site in the year 2000 (Alpert and Kishcha, 2008; Stanhill and Cohen, 2001). We extended these studies by comparing the PI change from 1960 to 1990 of individual sites, calculated from a 10 times finer resolved population data set, against the SSR trend from 1964 to 1989 (i.e. the same period as in Alpert and Kishcha, 2008). The PI of a GEBA site is the distance-weighted mean of the population distribution within a radius of $500 \mathrm{~km}$ from the site. Distance weights are used to mimic a simple aerosol diffusion model.

Overall we found positive but insignificant correlation coefficients between the SSR trends and the change in PIs from 1960 to 1990 for the GEBA sites in Europe and Japan. Note that an urbanization impact would require a negative correlation. We conclude for the groups of sites in Europe and Japan that urbanization measured as a population increase in the surrounding of the site does not go hand in hand with a stronger negative SSR trend. This is in line with the findings of Stanhill and Cohen (2009) for the SSR measurement sites in Israel, where SSR trends during the dimming period and the respective rates of change in population were found to be unrelated. Our findings for Europe and Japan are also consistent with the study by Wang et al. (2014), who showed that urbanization (inferred from night-time light data) had no discernible impact on the SSR trends. 

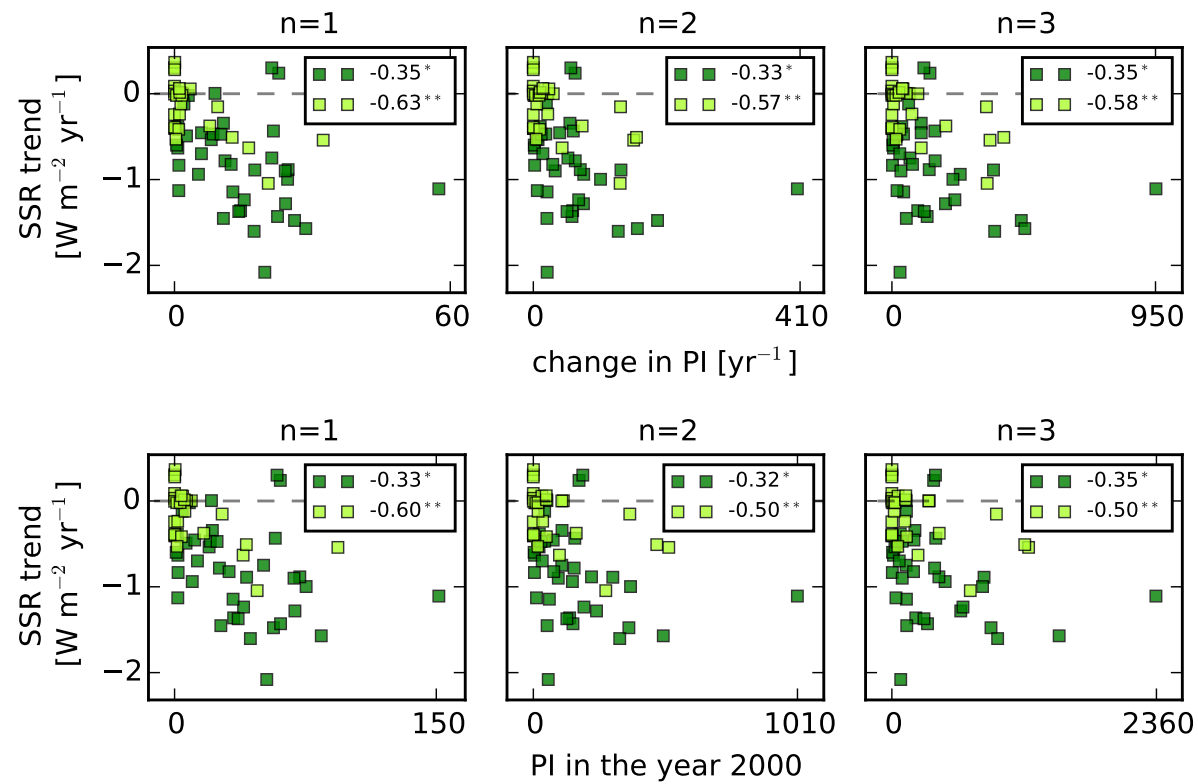

Figure 5. Further split of the 65 Asian sites into the 39 Chinese (darker green) and 26 non-Chinese, mostly Russian (22) sites (lighter green). The first (second) row of plots shows the same as the first row in Fig. 3 (Fig. 4). The corresponding correlation coefficients are shown in the boxes. Significant correlations at the $5 \%(1 \%)$ level are indicated with an ***).

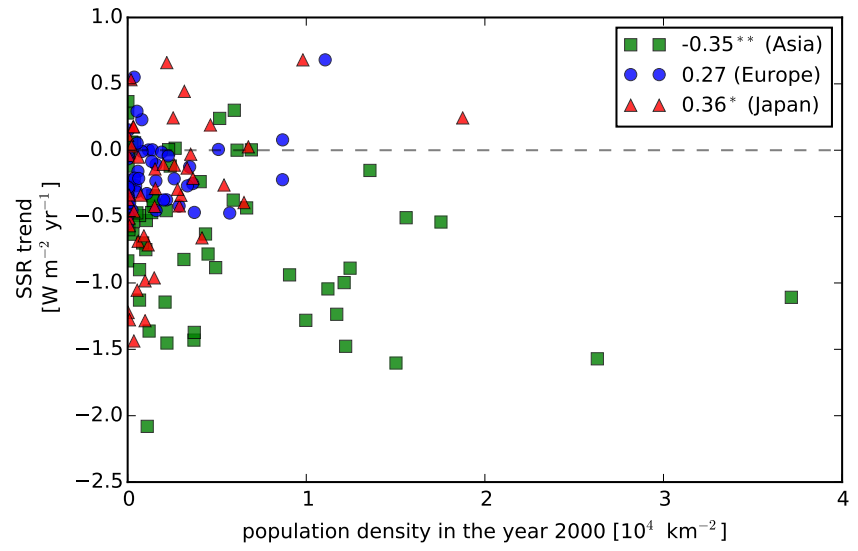

Figure 6. Unweighted population density in the $0.08^{\circ}$ cell surrounding the sites in the year 2000 vs. the SSR trend from 1964 to 1989 of the sites in the three regions. Significant correlations at the $5 \%(1 \%)$ are indicated with ${ }^{*}\left({ }^{* *}\right)$. The dashed line separates positive and negative SSR trends.

On the contrary our findings for the 92 sites in Europe and Japan are not consistent with Alpert and Kishcha (2008), who exclusively focused on population density of the year 2000 as an urbanization indicator. The present study does not discern an urbanization impact for the sites in Europe and Japan, irrespective of the choice of indicators: PI changes from 1960 to 1990 , absolute PI in the year 2000 or population density in the year 2000. In contrast to Alpert and Kishcha (2008) we furthermore avoided aggregating sites into two single urban and rural groups, as this may induce spurious biases in their average SSR trends due to the different geographical distribution of the sites in the two groups (Wang et al., 2014).

Unlike for the sites in Europe and Japan, the present study does not preclude an urbanization impact for the sites in Asia. The correlations for the 39 Chinese and 26 non-Chinese, mostly Russian sites are negative and significant at the $5 \%$ level. The correlation coefficients (SSR trend vs. PI change from 1960 to 1990) for the non-Chinese sites in Asia are the largest found and range from -0.5 to -0.63 . For the Chinese sites the correlation coefficients range between -0.3 to -0.34 , while the average SSR trends there are stronger than in the group of non-Chinese sites in Asia.

Our findings suggest that changes in PI from 1960 to 1990 or absolute levels of PI in the year 2000 (and hence any crude use of population density) provide no convincing evidence that global dimming is mostly an urban and hence local phenomenon for the sites in Europe and Japan. However, an urbanization effect for the sites in China and, particularly, for those in Russia cannot be ruled out. Particularly for the Chinese GEBA sites complementary studies are necessary, given potential data inhomogeneity and instrumentation issues which were recently reported by Wang et al. (2015).

Further research is required to clarify the importance of urbanization for SSR trends during the dimming period. 


\section{Data availability}

The SSR data and the population data used in this study are publicly accessible. They were taken from www.geba.ethz.ch and ftp://ftp.pbl.nl/hyde/, respectively.

Acknowledgements. We thank Gunnar Myhre and Atsumu Ohmura for the discussions that motivated this study. We also thank Christoph Schär for continuous support. Katsumasa Tanaka is funded by a Marie Curie Intra-European Fellowship within the 7th European Community Framework Programme (Proposal No. 255568 under FP7-PEOPLE-2009-IEF).

Edited by: S. Kloster

\section{References}

Alpert, P. and Kishcha, P.: Quantification of the effect of urbanization on solar dimming, Geophys. Res. Lett., 35, L08801, doi:10.1029/2007GL033012, 2008.

Gilgen, H. and Ohmura, A.: The global energy balance archive, B. Am. Meteorol. Soc., 80, 831-850, doi:10.1175/15200477(1999)080<0831:TGEBA>2.0.CO;2, 1999.

Gilgen, H., Wild, M., and Ohmura, A.: Means and trends of shortwave irradiance at the surface estimated from global energy balance archive data, J. Climate, 114, 2042-2061, doi:10.1175/1520-0442-11.8.2042, 1998.

Klein Goldewijk, K., Beusen, A., and Janssen, P.: Long-term dynamic modeling of global population and built-up area in a spatially explicit way: HYDE 3.1, Holocene, 20, 565-573, doi:10.1177/0959683609356587, 2010.

Liepert, B. G.: Observed reductions of surface solar radiation at sites in the United States and worldwide from 1961 to 1990, Geophys. Res. Lett., 29, 61.1-61.4, doi:10.1029/2002GL014910, 2002.

Liley, J. B.: New Zealand dimming and brightening, J. Geophys. Res., 114, D00D10, doi:10.1029/2008JD011401, 2009.

Norris, J. R. and Wild, M.: Trends in aerosol radiative effects over Europe inferred from observed cloud cover, solar "dimming," and solar "brightening”, J. Geophys. Res., 112, D08214, doi:10.1029/2006JD007794, 2007.
Ohmura, A.: Observed decadal variations in surface solar radiation and their causes, J. Geophys. Res., 114, D00D05, doi:10.1029/2008JD011290, 2009.

Ohmura, A. and Lang, H.: Secular variation of global radiation in Europe, in: IRS'88: Current Problems in Atmospheric Radiation, edited by: Lenoble, J. and Geleyn, J.-F., A. Deepak, Hampton, VA, 298-301, 1989.

Skeie, R. B., Berntsen, T. K., Myhre, G., Tanaka, K., Kvalevåg, M. M., and Hoyle, C. R.: Anthropogenic radiative forcing time series from pre-industrial times until 2010, Atmos. Chem. Phys., 11, 11827-11857, doi:10.5194/acp-11-11827-2011, 2011.

Stanhill, G. and Cohen, S.: Global dimming: a review of the evidence for a widespread and significant reduction in global radiation with discussion of its probable causes and possible agricultural consequences, Agr. Forest Meteorol., 107, 255-278, doi:10.1016/S0168-1923(00)00241-0, 2001.

Stanhill, G. and Cohen, S.: Solar radiation changes in Japan during the 20th century: evidence from sunshine duration measurements, J. Meteorol. Soc. Jpn., 86, 57-67, doi:10.2151/jmsj.86.57, 2008.

Stanhill, G. and Cohen, S.: Is solar dimming global or urban? Evidence from measurements in Israel between 1954 and 2007 J. Geophys. Res., 114, 2156-2202, doi:10.1029/2009JD011976, 2009.

Wang, K., Ma, Q., Wang, X., and Wild, M.: Urban impacts on mean and trend of surface incident solar radiation, Geophys. Res. Lett., 41, 4664-4668, doi:10.1002/2014GL060201, 2014.

Wang, K., Ma, Q., Li, Z., and Wang, J.: Decadal variability of surface incident solar radiation over China: Observations, satellite retrievals, and reanalyses, Geophys. Res. Atmos., 120, 65006514, doi:10.1002/2015JD023420, 2015.

Wild, M.: Global dimming and brightening: a review, J. Geophys. Res., 114, D00D16, doi:10.1029/2008JD011470, 2009.

Wild, M., Gilgen, H., Roesch, A., Ohmura, A., Long, C. N., Dutton, E. G., Forgan, B., Kallis, A., Russak, V., and Tsevetkov, A.: From dimming to brightening: decadal changes in solar radiation at Earth's surface, Science, 308, 847-850, doi:10.1126/science.1103215, 2005. 